\title{
Software-Directed Register Deallocation for Simultaneous Multithreaded Processors
}

\author{
Jack L. Lo, Sujay S. Parekh, Susan J. Eggers, Henry M. Levy, and Dean M. Tullsen* \\ Dept. of Computer Science and Engineering \\ Box 352350 \\ University of Washington \\ Seattle, WA 98195-2350 \\ jlo@cs.washington.edu \\ *Dept. of Computer Science and Engineering \\ University of California, San Diego \\ 9500 Gilman Drive \\ La Jolla, CA 92093-0114
}

University of Washington Technical Report \#UW-CSE-97-12-01

\begin{abstract}
This paper proposes and evaluates software techniques that increase register file utilization for simultaneous multithreading (SMT) processors. SMT processors require large register files to hold multiple thread contexts that can issue instructions, out of order, every cycle. By supporting better inter-thread sharing and management of physical registers, an SMT processor can reduce the number of registers required and can improve performance for a given register file size.

Our techniques specifically target register deallocation. While out-of-order processors with register renaming are effective at knowing when a new physical register must be allocated, they are limited in knowing when physical registers can be deallocated. We propose architectural extensions that permit the compiler and operating system to (1) free registers immediately upon their last use, and (2) free registers allocated to idle thread contexts. Our results, based on detailed instruction-level simulations of an SMT processor, show that these techniques can increase performance significantly for register-intensive, multithreaded programs.
\end{abstract}

\section{Introduction}

Simultaneous multithreading (SMT) is a high-performance architectural technique that substantially improves processor performance by executing multiple instructions from multiple threads every cycle. By dynamically sharing processor resources among threads, SMT increases functional unit utilization, thereby boosting both instruction throughput for multiprogrammed workloads and application speedup for multithreaded programs [5].

Previous research has looked at the performance potential of SMT [24], as well as several 
portions of its design, including instruction fetch mechanisms and cache organization [23][13]. This paper focuses on another specific design area that impacts SMT's cost-effectiveness: the organization and utilization of its register file. SMT raises a difficult tradeoff for register file design: while a large register file is required to service the architectural and renaming register needs of the multiple thread contexts, smaller register files provide faster access times. Therefore, an SMT processor needs to use its register resources efficiently in order to optimize both die area and performance.

In this paper, we propose and evaluate software techniques that increase register utilization, permitting a smaller, faster register file, while still satisfying the processor's need to support multiple threads. Our techniques involve coordination between the operating system, the compiler, and the low-level register renaming hardware to provide more effective register use for both single-threaded and multithreaded programs. The result is improved performance for a given number of hardware contexts and the ability to handle more contexts with a given number of registers. For example, our experiments indicate that an 8-context SMT processor with 264 physical registers, managed with the techniques we present, can attain performance comparable to a processor with 352 physical registers.

Our techniques focus on supporting the effective sharing of registers in an SMT processor, using register renaming to permit multiple threads to share a single global register file. In this way, one thread with high register pressure can benefit when other threads have low register demands. Unfortunately, existing register renaming techniques cannot fully exploit the potential of a shared register file. In particular, while existing hardware is effective at allocating physical registers, it has only limited ability to identify register deallocation points; therefore hardware must free registers conservatively, possibly wasting registers that could be better utilized.

We propose software support to expedite the deallocation of two types of dead registers: (1) registers allocated to idle hardware contexts, and (2) registers in active contexts whose last use has already retired. In the first case, when a thread terminates execution on a multithreaded architecture, its hardware context becomes idle if no threads are waiting to run. While the registers allocated to the terminated thread are dead, they are not freed in practice, because hardware register deallocation only occurs when registers in a new, active thread are mapped. This causes a potentially-shared SMT register file to behave like a partitioned collection of perthread registers. Our experiments show that by notifying the hardware of OS scheduling decisions, performance with a register file of size 264 is boosted by more than 3 times when 2 or 4 threads are running, so that it is comparable to a processor with 352 registers.

To address the second type of dead registers, those in active threads, we investigate five mechanisms that allow the compiler to communicate last-use information to the processor, so that the renaming hardware can deallocate registers more aggressively. Without this information, the hardware must conservatively deallocate registers only after they are redefined. Simulation results indicate that these mechanisms can reduce register deallocation inefficiencies; in particular, on small register files, the best of the schemes attains speedups of up to 2.5 for some applications, and 1.6 on average. All the register deallocation schemes could benefit any out-of-order processor, not just SMT.

The remainder of this paper is organized as follows. Section 2 briefly summarizes the SMT architecture and register renaming inefficiencies. Our experimental methodology is described in Section 3. Section 4 describes the OS and compiler support that we use to improve register usage. We discuss related work in Section 5 and offer concluding remarks in Section 6. 


\section{Simultaneous Multithreading}

Our SMT processor model is similar to that used in previous studies: an eight-wide, out-oforder processor with hardware contexts for eight threads. On every cycle four instructions are fetched from each of two threads. The fetch unit favors high throughput threads, selecting the two threads that have the fewest instructions waiting to be executed. After fetching, instructions are decoded, their registers are renamed, and they are inserted into either the integer or floating point instruction queues. When their operands become available, instructions (from any thread) are issued to the functional units for execution. Finally, instructions are retired in per-thread order.

Most components of an SMT processor are an integral part of any dynamically-scheduled, wide-issue superscalar. Instruction scheduling is an important case in point: instructions are issued after their operands have been calculated or loaded from memory, without regard to thread; the register renaming hardware eliminates inter-thread register name conflicts by mapping thread-specific architectural registers onto the processor's physical registers.

The major additions to a conventional superscalar are the instruction fetch unit mentioned above and several per-thread mechanisms, such as program counters, return stacks, retirement and trap logic, and identifiers in the TLB and branch target buffer. The register file contains register state for all processor-resident threads and consequently requires two additional pipeline stages for accessing it (one each for reading and writing). (See [23] for more details.)

\subsection{Register Renaming and the Register Deallocation Problem}

Register renaming eliminates false (output and anti-) dependences that are introduced when the compiler's register allocator assigns an arbitrary number of pseudo-registers to the limited number of architectural registers in the instruction set architecture. Dependences are broken by dynamically aliasing each defined architectural register to a different physical register, enabling formerly dependent instructions to be executed in parallel.

SMT assumes a register mapping scheme similar to that used in the DEC 21264 [8] and MIPS R10000 [27]. The register renaming hardware is responsible for three primary functions: (1) physical register allocation, (2) register operand renaming, and (3) register deallocation. Physical register allocation occurs on demand. When an instruction defines an architectural register, a mapping is created from the architectural register to an available physical register and is entered into the mapping table. If no registers are available, instruction fetching stalls. To rename a register operand, the renaming hardware locates its architectural-to-physical mapping in the mapping table and aliases it to its physical number. Register deallocation works in conjunction with instruction retirement. An active list keeps track of all uncommitted instructions in per-thread program order. As instructions retire, their physical registers are deallocated and become available for reallocation.

Renaming hardware handles physical register allocation and renaming rather effectively, but fails to manage deallocation efficiently. A register is dead and could be deallocated once its last use commits. The hardware, however, cannot identify the last uses of registers, because it has no knowledge of register lifetimes. Consequently, hardware can only safely deallocate a physical register when it commits another instruction that redefines its associated architectural register, as shown in Figure 1.

\subsection{Physical Register Organization and the Register Deallocation Problem}

In fine-grained multithreaded architectures like the Tera [1], each hardware context includes 


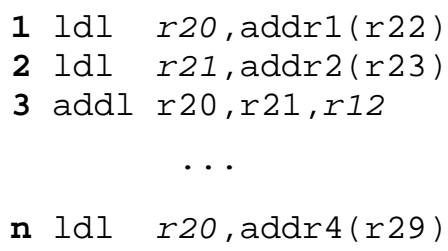

(a)

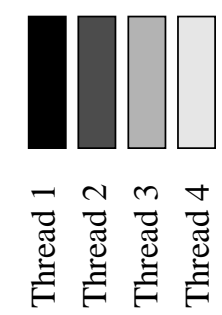

Figure 1: This example illustrates the inability of the renaming hardware to efficiently deallocate the physical register for $\mathrm{r} 20$. (The destination registers are italicized). Instruction 1 defines $\mathrm{r} 20$, creating a mapping to a physical register, say P1. Instruction 3 is the last use of r20. However, P1 cannot be freed until r 20 is redefined in instruction $\mathrm{n}$. In the meantime, several instructions and potentially a large number of cycles can pass between the last use of P1 (r20) and its deallocation. (b)

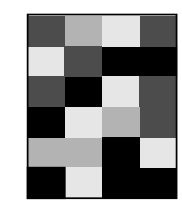

(c)

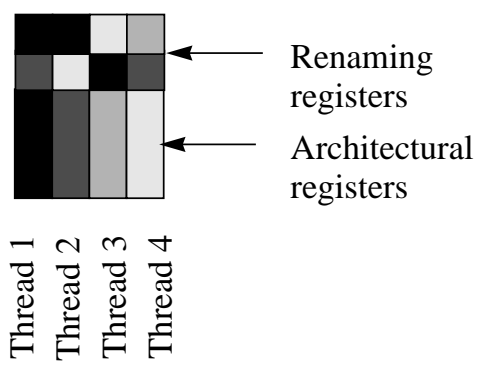

Figure 2: Logical register file configurations: (a) is a Tera-style, partitioned register file; (b) is an SMT register file in which all threads share a common pool of physical registers; (c) is an SMT register file, given current register deallocation schemes: each hardware context has dedicated physical registers for the ISA-defined architectural registers and only the renaming registers are shared across all contexts.

a register file for one thread, and a thread only accesses registers from its own context, as shown in Figure 2a. ${ }^{1}$ In contrast, in an SMT processor, a single register file can be shared among all contexts (Figure 2b). We call this organization FSR ( Fully-Shared Registers), because the register file is structured as a single pool of physical registers and holds the state of all resident threads. SMT's register renaming hardware is essentially an extension of the register mapping scheme to multiple contexts. Threads name architectural registers from their own context, and the renaming hardware maps these thread-private, architectural registers to the pool of threadindependent physical registers. Register renaming thus provides a transparent mechanism for sharing the register pool.

Although an SMT processor is best utilized when all hardware contexts are busy, some contexts may occasionally be idle. To maximize performance, no physical registers should be allocated to idle contexts; instead, all physical registers should be shared by the active threads. However, with existing register deallocation schemes, when a thread terminates, its architectural registers remain allocated in the processor until they are redefined by a new thread executing in the context. Consequently, the FSR organization behaves more like a partitioned file, as shown in Figure 2c. (We call this partitioned organization PASR, for Private Architectural and Shared Renaming registers.) Most ISAs have 32 architectural registers; consequently, thirty-two physical registers must be dedicated to each context in a PASR scheme. So, for example, on an eight-context SMT with 352 registers, only 96 (352-8*32) physical registers are available for sharing among the active threads. 


\section{Methodology for the Experiments}

We have defined several register file management techniques devised to compensate for conservative register deallocation, and evaluated them using instruction-level simulation of applications from the SPEC 95 [20] and SPLASH-2 [26] benchmark suites (Table 1). The SUIF compiler [9] automatically parallelized the SPEC benchmarks into multithreaded C code; the SPLASH-2 programs were already explicitly parallelized by the programmer. All programs were compiled with the Multiflow trace-scheduling compiler [14] into DEC Alpha object files. (Multiflow generates high-quality code, using aggressive static scheduling for wide issue, loop unrolling, and other ILP-exposing optimizations.) The object files were then linked with our

\begin{tabular}{|c|c|c|c|}
\hline & Application & data set & instructions simulated \\
\hline \multirow[t]{7}{*}{ SPEC 95 FP } & applu & $33 \times 33 \times 33$ array, 2 iterations & $271.9 \mathrm{M}$ \\
\hline & hydro2d & 2 iterations & $473.5 \mathrm{M}$ \\
\hline & mgrid & $64 \times 64 \times 64$ grid, 1 iteration & $3.193 \mathrm{~B}$ \\
\hline & su2cor & $16 \times 16 \times 16 \times 16$, vector length 4096,2 iterations & $5.356 \mathrm{~B}$ \\
\hline & swim & $512 \times 512$ grid, 10 iterations & $419.1 \mathrm{M}$ \\
\hline & tomcatv & $513 \times 513$ array, 5 iterations & $189.1 \mathrm{M}$ \\
\hline & turb3d & $64 \times 64 \times 64$ array, 1 timestep & $1.941 \mathrm{~B}$ \\
\hline \multirow[t]{5}{*}{ SPLASH 2} & $\mathrm{fft}$ & $64 \mathrm{~K}$ data points & $32.0 \mathrm{M}$ \\
\hline & $\mathrm{LU}$ & $512 \times 512$ matrix & $431.2 \mathrm{M}$ \\
\hline & radix & $256 \mathrm{~K}$ keys, radix 1024,524288 max key value & $5.8 \mathrm{M}$ \\
\hline & water-nsquared & 512 molecules, 3 timesteps & $869.9 \mathrm{M}$ \\
\hline & water-spatial & 512 molecules, 3 timesteps & $783.5 \mathrm{M}$ \\
\hline
\end{tabular}

Table 1: Benchmarks used in this study. For the SPEC95 applications, our data sets are the same size as the SPEC reference set, but we have reduced the number of iterations because of the length of simulation time.

versions of the ANL [2] and SUIF runtime libraries to create executables.

Our SMT simulator processes unmodified Alpha executables and uses emulation-based, instruction-level simulation to model in detail the processor pipelines, hardware support for outof-order execution, and the entire memory hierarchy, including the TLBs (128 entries each for instruction and data TLBs), cache behavior, and bank and bus contention. The memory hierarchy in our processor consists of two levels of cache, with sizes, latencies, and bandwidth characteristics, as shown in Table 2. Because register file management is affected by memory

\begin{tabular}{|l||c|c|c|}
\hline & L1 I-cache & L1 D-cache & L2 cache \\
\hline Size (bytes) & $128 \mathrm{~K} / 32 \mathrm{~K}$ & $128 \mathrm{~K} / 32 \mathrm{~K}$ & $16 \mathrm{M} / 2 \mathrm{M}$ \\
\hline Associativity & two-way & two-way & direct- mapped \\
\hline Line size (bytes) & 64 & 64 & 64 \\
\hline Banks & 4 & 4 & 1 \\
\hline Accesses/cycle & 2 & 2 & $1 / 2$ \\
\hline Cache fill time (cycles) & 2 & 2 & 4 \\
\hline Latency to next level & 10 & 10 & 68 \\
\hline
\end{tabular}

Table 2: Configuration and latency parameters of the SMT cache hierarchies used in this study. 
latencies, we experimented with two different memory hierarchies. The larger memory configuration represents a probable SMT memory hierarchy for machines in production approximately 3 years in the future. The smaller configuration serves two purposes: (1) it models today's memory hierarchies, as well as those of tomorrow's low-cost processors, such as multimedia co-processors, and (2) it provides a more appropriate ratio between data set and cache size, modeling programs with larger data sets or data sets with less data locality than those in our benchmarks [19].

We also examined a variety of register file sizes, ranging between 264 and 352, to gauge the sensitivity of the register file management techniques to register size. With more than 352 registers, other processor resources, such as the instruction queues, become performance bottlenecks. At the low end, at least 256 registers are required to hold the architectural registers for all eight contexts, ${ }^{2}$ and we provide an additional 8 renaming registers for a total of 264 . Smaller register files are attractive for several reasons. First, they have a shorter access time; this advantage could be used either to decrease the cycle time (if register file access is on the critical path) or to eliminate the extra stages we allow for register reading and writing. Second, they take up less area. Register files in current processors occupy a negligible portion (roughly 1\%) of the chip area, but a large, multi-ported SMT register file could raise that to around $10 \%$, an area allocation that might not be acceptable. Third, smaller register files consume less power.

For branch prediction, we used a McFarling-style hybrid predictor with a 256-entry, 4-way set-associative branch target buffer, and a hybrid predictor (8k entries) that selects between a global history predictor (13 history bits) and a local predictor (a $2 \mathrm{k}$-entry local history table that indexes into a 4k-entry, 2-bit local prediction table) [16].

Because of the length of the simulations, we limited our detailed simulation results to the parallel computation portion of the applications (the norm for simulating parallel applications). For the initialization phases of the applications, we used a fast simulation mode that warmed the caches, and then turned on the detailed simulation mode once the main computation phases were reached.

\section{Techniques for improving register file management}

Despite its flexible organization, an SMT register file will be underutilized, because renaming hardware fails to deallocate dead registers promptly. In this section, we describe communication mechanisms that allow the operating system and the compiler to assist the renaming hardware with register deallocation, by identifying dead registers that belong to both idle and active contexts.

\subsection{Operating system support for dead-register deallocation}

As explained in Section 2.2, when an executing thread terminates, the thread's physical registers remain allocated. Consequently, active threads cannot access these registers, causing a fully-shared register file (FSR) to behave like one in which most of the registers are partitioned by context (PASR).

After a thread terminates, the operating system decides what to schedule on the newlyavailable hardware context. There are three options, each of which has a different implication 
for register deallocation:

1. Idle contexts: If there are no new threads to run, the context will be idle. The terminated thread's physical registers could be deallocated, so that they become available to active threads.

2. Switching to a new thread: Physical registers for a new thread's architectural registers are normally allocated when it begins execution. A more efficient scheme would free the terminated threads's physical registers, allocating physical registers to the new thread on demand. Unallocated physical registers would then be available to other contexts.

3. Switching to a swapped-out thread: Context switch code loads the register state of the new thread. As these load instructions retire, physical registers used by the terminated thread are deallocated.

All three scenarios present an opportunity to deallocate a terminated thread's physical registers early. We propose a privileged, context deallocation instruction (CDI) that triggers physical register deallocation for a thread. The operating system scheduler would execute the instruction in the context of the terminated thread. In response, the renaming hardware would free the terminating thread's physical registers when the instruction retires.

Three tasks must be performed to handle the context deallocation instruction: creating a new map table, invalidating the context's register mappings, and returning the registers to the free list. When a CDI enters the pipeline, the current map table is saved and a new map table with no valid entries is created; the saved map table identifies the physical registers that should be deallocated, while the new table will hold subsequent register mappings. Once the CDI retires, the saved map is traversed, and all mapped physical registers are returned to the free list. Finally, all entries in the saved map are invalidated. If the CDI is executed on a wrong-path and consequently gets squashed, both the new and saved map tables are thrown away.

Much of the hardware required for these three tasks already exists in out-of-order processors with register mapping. When a branch enters the pipeline, a copy of the map table is created; when the branch is resolved, one of the map tables is invalidated, depending on whether the speculation was correct. If instructions must be squashed, the renaming hardware traverses the active list (or some other structure that identifies physical registers) to determine which physical registers should be returned to the free list. Although the CDI adds a small amount of logic to existing renaming hardware, it allows the SMT register file to behave as a true FSR register file, instead of a PASR by deallocating registers more promptly.

\section{Experimental results}

To evaluate the performance of the fully-shared register organization (FSR), we varied the number of active threads and register set sizes, and compared it to PASR with identical configurations. We modeled an OS scheduler that frees all physical registers for terminated threads, by making all physical registers available when a parallel application began execution.

The results of this comparison are shown in Figure 3. With PASR (Figure 3a), only renaming registers are shared among threads. Execution time therefore was greater for smaller register files and larger numbers of threads, as more threads competed for fewer registers. FSR, shown in Figure 3b, was less sensitive to both parameters. In fact, the smaller register files had the same performance as larger ones when few threads were executing, because registers were not tied up by idle contexts. Except for the smallest configuration, FSR performance was stable with varying numbers of threads, because the parallelism provided by additional threads overcame the increased competition for registers; only the 264-register file had a performance 
(a) PASR

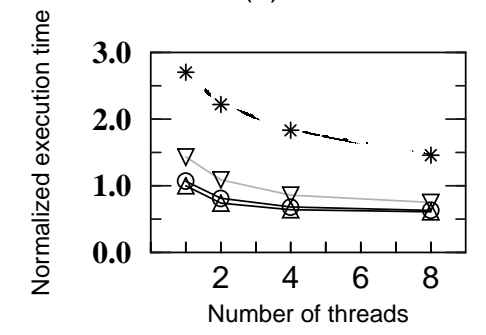

(b) FSR

Number of registers

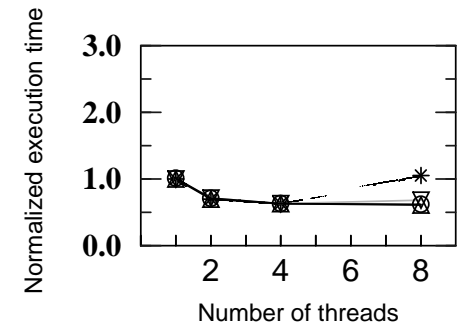

Figure 3: Execution time for FSR and PASR with the larger memory hierarchy. Each register file organization is normalized to its 352 register, 1 thread execution. Results with the smaller SMT memory hierarchy had identical trends.
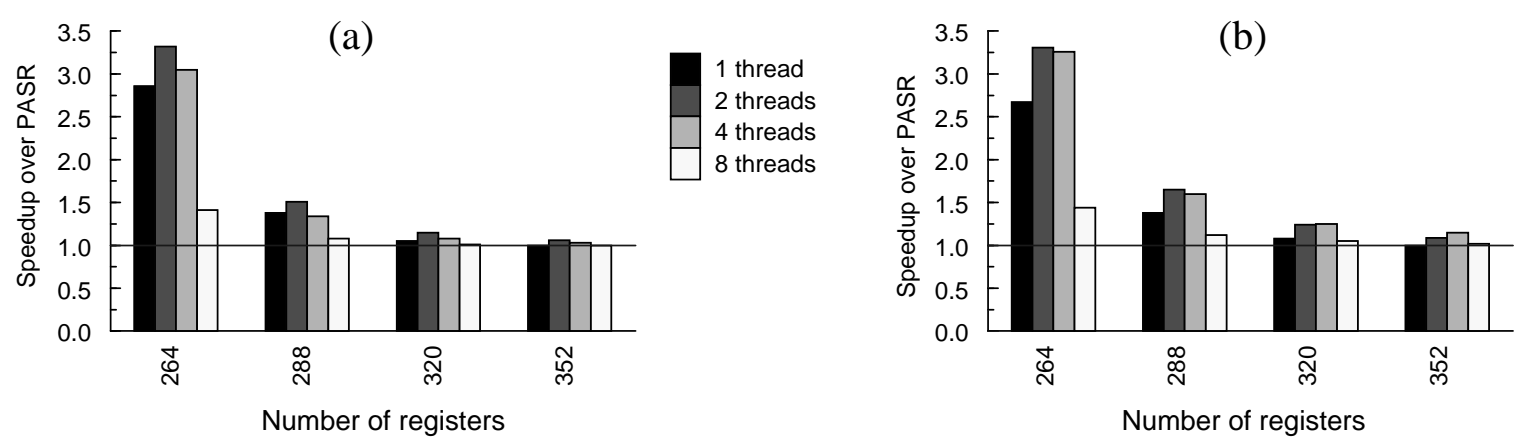

Figure 4: FSR speedups over PASR for the larger (a) and smaller (b) memory hierarchies, at different register file sizes.

sweet spot.

The speedups in Figure 4 show that FSR equals or surpasses PASR for all register file sizes and numbers of threads. FSR provides the greatest benefits when it has more registers to share (several idle contexts) and PASR has fewer (small register files). For example, with 320 registers and 4 idle contexts (4 threads), FSR outperformed PASR by $8 \%$, averaged over all applications. With only 288 or 264 registers, FSR's advantage grew to $34 \%$ and $205 \%$, and with 6 idle contexts (and 320 registers) to 15\%. Taking both factors into account (288/264 registers, 6 idle contexts), FSR outperformed PASR by $51 \% / 232 \%$. Only when all contexts were active were FSR and PASR comparable; in this case the architectural state for all threads is resident in both schemes.

FSR has a larger performance edge with smaller cache hierarchies, because hiding the longer memory latencies requires more in-flight instructions, and therefore more outstanding registers. This suggests that efficient register management is particularly important on memory-intensive workloads or applications with relatively poor data locality.

In summary, the results illustrate that partitioning a multithreaded register file (PASR) restricts its ability to expose parallelism. Operating system support for deallocating registers in idle contexts, which enables the register file to be fully shared across all threads (FSR), both improves performance, and makes it less dependent on the size of the register file and the number of active threads.

\subsection{Compiler support for dead-register allocation}

As previously described, hardware register deallocation is inefficient, because the hardware 
has knowledge only of a register's redefinition, not its last use. Although the compiler can identify the last use of a register, it currently has no means for communicating this information to the hardware.

In this section, we describe and evaluate several mechanisms that allow the compiler to convey register last-use information to the hardware, and show that they improve register utilization on SMT processors with an FSR register file organization. The proposed mechanisms are either new instructions or fields in existing instructions that direct the renaming hardware to free physical registers.

\begin{tabular}{|c|c|c|c|c|c|c|c|c|c|c|c|c|c|c|c|c|}
\hline \multirow{2}{*}{$\begin{array}{l}\text { Number } \\
\text { of } \\
\text { registers }\end{array}$} & \multicolumn{8}{|c|}{ integer } & \multicolumn{8}{|c|}{ FP } \\
\hline & applu & hydro2d & swim & tomcatv & fft & $\mathbf{L U}$ & radix & water-n & applu & hydro2d & swim & tomcatv & fft & $\mathbf{L U}$ & radix & water-n \\
\hline \multicolumn{17}{|c|}{ Large Cache Hierarchy } \\
\hline 352 & 3.1 & 0.1 & 0.0 & 0.0 & 3.8 & 0.0 & 0.4 & 0.0 & 27.7 & 6.3 & 0.0 & 18.0 & 0.0 & 0.0 & 0.0 & 0.1 \\
\hline 320 & 3.1 & 0.7 & 0.1 & 0.0 & 16.1 & 0.1 & 36.9 & 0.1 & 40.2 & 17.7 & 0.1 & 20.3 & 0.0 & 0.0 & 0.0 & 0.6 \\
\hline 288 & 1.9 & 1.6 & 0.4 & 0.0 & 21.5 & 0.3 & 75.9 & 0.3 & 64.0 & 521 & 0.5 & 58.4 & 0.5 & 0.0 & 0.0 & 14.1 \\
\hline 264 & 1.5 & 2.2 & 21.9 & 0.0 & 58.2 & 2.2 & 91.1 & 0.6 & 87.8 & 83.0 & 0.6 & 90.1 & 8.1 & 0.2 & 0.0 & 73.9 \\
\hline
\end{tabular}

\begin{tabular}{|c||c|c|c|c|c|c|c|c||c|c|c|c|c|c|c|c|}
\hline \multicolumn{11}{|c|}{ Small Cache Hierarchy } \\
\hline 352 & 3.4 & 1.0 & 0.4 & 0.0 & 8.4 & 1.3 & $\mathbf{1 2 . 0}$ & 0.0 & $\mathbf{3 2 . 3}$ & $\mathbf{3 4 . 4}$ & 5.3 & $\mathbf{4 5 . 7}$ & 0.5 & 1.7 & 0.0 & 0.1 \\
\hline 320 & 3.5 & 1.5 & 1.5 & 0.0 & $\mathbf{1 6 . 2}$ & 3.3 & $\mathbf{4 9 . 1}$ & 0.2 & $\mathbf{4 4 . 6}$ & $\mathbf{4 8 . 0}$ & 8.6 & $\mathbf{6 0 . 2}$ & 1.4 & 3.5 & 0.0 & 0.6 \\
\hline 288 & 2.6 & 2.1 & 5.3 & 0.0 & $\mathbf{2 5 . 2}$ & $\mathbf{1 0 . 5}$ & $\mathbf{8 3 . 5}$ & 0.3 & $\mathbf{6 7 . 5}$ & $\mathbf{7 0 . 1}$ & $\mathbf{1 3 . 2}$ & $\mathbf{7 9 . 8}$ & 5.3 & 5.5 & 0.0 & $\mathbf{1 7 . 0}$ \\
\hline 264 & 2.2 & 2.5 & $\mathbf{4 4 . 2}$ & 0.2 & $\mathbf{6 4 . 9}$ & $\mathbf{2 2 . 7}$ & $\mathbf{9 3 . 4}$ & 0.7 & $\mathbf{8 8 . 1}$ & $\mathbf{8 7 . 2}$ & 2.7 & $\mathbf{9 4 . 0}$ & 8.5 & 6.7 & 0.0 & $\mathbf{7 4 . 7}$ \\
\hline
\end{tabular}

Table 3: Frequency (percentage of total execution cycles) that no registers were available when executing 8 threads. Bold entries (frequencies over 10\%) represent severe stalling due to insufficient registers.

\begin{tabular}{|c|c|c|c|c|c|c|c|c|c|c|c|c|c|c|c|c|}
\hline \multirow{2}{*}{$\begin{array}{l}\text { Number } \\
\text { of } \\
\text { registers }\end{array}$} & \multicolumn{8}{|c|}{ integer } & \multicolumn{8}{|c|}{ FP } \\
\hline & applu & hydro2d & swim & tomcatv & fft & LU & radix & water-n & applu & hydro2d & swim & tomcatv & fft & $\mathbf{L U}$ & radix & water-n \\
\hline \multicolumn{17}{|c|}{ Large Cache Hierarchy } \\
\hline 352 & 74 & 69 & 29 & 52 & 135 & 110 & 312 & 168 & 120 & 153 & 73 & 136 & 68 & 42 & 48 & 199 \\
\hline 320 & 74 & 68 & 29 & 53 & 134 & 110 & 311 & 168 & 120 & 153 & 73 & 136 & 68 & 42 & 36 & 199 \\
\hline 288 & 75 & 64 & 29 & 52 & 132 & 110 & 301 & 168 & 117 & 152 & 73 & 135 & 67 & 42 & 48 & 199 \\
\hline 264 & 77 & 57 & 29 & 51 & 140 & 110 & 211 & 168 & 111 & 150 & 73 & 133 & 71 & 42 & 48 & 198 \\
\hline
\end{tabular}

\begin{tabular}{|l||l|l|l|l|l|l|l|l||l|l|l|l|l|l|l|l|}
\hline \multicolumn{10}{|c|}{ Small Cache Hierarchy } \\
\hline 352 & 74 & 62 & 30 & 54 & 132 & 147 & $\mathbf{2 6 5}$ & 168 & $\mathbf{1 2 1}$ & $\mathbf{1 5 8}$ & 77 & $\mathbf{1 4 1}$ & 68 & 88 & 24 & 199 \\
\hline 320 & 74 & 61 & 30 & 55 & $\mathbf{1 3 2}$ & 147 & $\mathbf{2 5 8}$ & 167 & $\mathbf{1 2 1}$ & $\mathbf{1 5 7}$ & 76 & $\mathbf{1 4 1}$ & 68 & 81 & 21 & 216 \\
\hline 288 & 74 & 58 & 30 & 55 & $\mathbf{1 3 1}$ & $\mathbf{1 1 0}$ & $\mathbf{2 3 1}$ & 168 & $\mathbf{1 1 8}$ & $\mathbf{1 5 5}$ & $\mathbf{7 6}$ & $\mathbf{1 3 9}$ & 69 & 50 & 44 & $\mathbf{1 9 9}$ \\
\hline 264 & 76 & 54 & $\mathbf{3 2}$ & 54 & $\mathbf{1 3 7}$ & $\mathbf{1 1 0}$ & $\mathbf{1 7 3}$ & 168 & $\mathbf{1 1 3}$ & $\mathbf{1 5 2}$ & 76 & $\mathbf{1 3 6}$ & 72 & 50 & 48 & $\mathbf{1 9 8}$ \\
\hline
\end{tabular}

Table 4: Average number of dead registers per cycle when executing 8 threads. Bold entries are those where no registers were available more than $10 \%$ of execution cycles.

First, however, we examine three factors that motivate the need for improved register deallocation: (1) how often physical registers are unavailable, (2) how many registers are dead each cycle, and (3) how many cycles pass between a register's last use and its redefinition, which we call the dead-register distance Register unavailability is the percentage of total execution cycles in which the processor runs out of physical registers (causing fetch stalls); it is a measure of the severity of the problem caused by current hardware register-deallocation mechanisms. The average number of dead registers each cycle indicates how many physical registers could be reused, and thus the potential for a compiler-based solution. Dead-register 


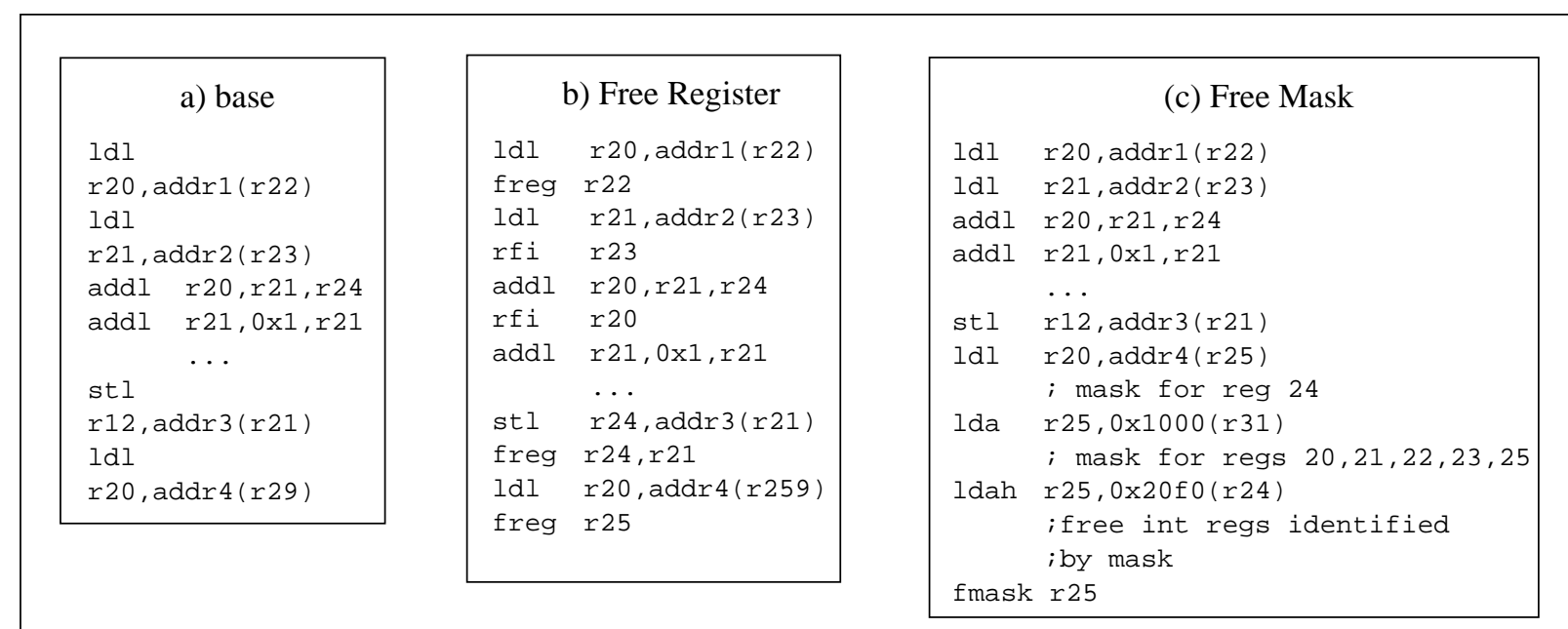

Figure 5: These code fragments illustrate the register freeing mechanisms: a) is the original code fragment; b) shows the Free Register instructions necessary to free registers r12 to r25, c) is the Free Mask instructions necessary to free the same registers

\begin{tabular}{|c||c|c|c|c|c|c|c|c||c|}
\hline $\begin{array}{c}\text { Number } \\
\text { of registers }\end{array}$ & applu & hydro2d & swim & tomcatv & fft & LU & radix & water-n & average \\
\hline int instrs & 57.6 & 59.1 & 32.3 & 67.2 & $\mathbf{3 0 . 7}$ & $\mathbf{5 6 . 9}$ & $\mathbf{2 7}$ & 32.7 & 47.2 \\
\hline int cycles & 214.6 & 155.4 & 27.8 & 225.7 & $\mathbf{8 9 . 9}$ & $\mathbf{8 5 . 6}$ & $\mathbf{8 0}$ & 215.4 & 125.5 \\
\hline FP instrs & $\mathbf{1 8 . 4}$ & $\mathbf{3 0 . 9}$ & 11.7 & $\mathbf{2 2 . 6}$ & 20.4 & 7.1 & & $\mathbf{3 2 . 7}$ & 18.5 \\
\hline FP cycles & $\mathbf{9 7 . 1}$ & $\mathbf{1 5 7 . 4}$ & 28.4 & $\mathbf{1 2 0 . 0}$ & 65.7 & 22.4 & & $\mathbf{1 3 3 . 7}$ & 81.8 \\
\hline
\end{tabular}

Table 5: Dead register distance for 264 registers and the smaller cache hierarchy. The data indicate that registers are frequently not deallocated until many cycles after their last use has retired. Figures for other register sizes were similar. Bold entries are those where no registers were available more than $10 \%$ of execution cycles.

distance measures the average number of cycles between the completion of an instruction that last uses a register and that register's deallocation; it is a rough estimate of the likely performance gain of a solution.

The data in Table 3 indicate that, while the projected SMT design (352 registers in an FSR file) is sufficient for most applications, smaller register files introduce bottlenecks, often severe, on many applications. (Register pressure was particularly high for integer registers in fft and radix, and for floating-point registers in applu, hydro2d, tomcatv, and water-n.) Applications also ran out of registers more frequently with smaller cache hierarchies. A closer examination reveals that in all cases where stalling due to insufficient registers was a problem (bold entries in Table 3), a huge number of registers were dead (shown in Table 4). Table 5 shows that if these dead registers had been freed, they could have been reallocated many instructions/cycles earlier. All this suggests that, if registers were managed more efficiently, performance could be recouped and even a 264-register FSR might be sufficient.

\section{Five compiler-based solutions}

Using dataflow analysis, the compiler can identify the last use of a register value. In this section, we evaluate five alternatives for communicating last-use information to the renaming hardware: 
1. Free Register Bit communicates last-use information to the hardware via dedicated instruction bits, with the dual benefits of immediately identifying last uses and requiring no instruction overhead. Although it is unlikely to be implemented, because most instruction sets do not have two unused bits, it can serve as an upper bound on performance improvements that can be attained with the compiler's static last-use information. To simulate Free Register Bit, we modified Multiflow to generate a table, indexed by the PC, that contains flags indicating whether either of an instruction's register operands were last uses. On each simulated instruction, the simulator performed a lookup in this table to determine whether register deallocation should occur when the instruction is retired.

2. Free Register is a more realistic implementation of Free Register Bit. Rather than specifying last uses in the instruction itself, it uses a separate instruction to specify one or two registers to be freed. Our compiler generates a Free Register instruction (an unused opcode in the Alpha ISA) immediately after any instruction containing a last register use (if the register is not also redefined by the same instruction). Like Free Register Bit, it frees registers as soon as possible, but with an additional cost in dynamic instruction overhead.

3. Free Mask is an instruction that can free up to 32 registers, and is used to deallocate dead registers over a large sequence of code, such as a basic block or a set of basic blocks. For our experiments, we inserted a Free Mask instruction at the end of each Multiflow trace. Rather than identifying dead registers in operand specifiers, the compiler generates a bit mask. In our particular implementation, the Free Mask instruction uses the lower 32-bits of a register as a mask to indicate which registers can be deallocated. The mask is generated and loaded into the register using a pair of lda and ldah instructions, each of which has a 16-bit immediate field. (The example in Figure 5 compares Free Register with Free Mask for a code fragment that frees integer registers 20 through 25.) Free Mask sacrifices the promptness of Free Register's deallocation for a reduction in instruction overhead.

4. Free Opcode is motivated by our observation that 10 opcodes were responsible for $70 \%$ of the dynamic instructions with last use bits set, indicating that most of the benefit of Free Register Bit could be obtained by providing special versions of those opcodes. In addition to executing their normal operation, the new instructions also specify that either the first, second, or both operands are last uses. In this paper, we use the 15 opcodes listed in Table 6, obtained by profiling Free Register Bit instruction frequencies on applu, hydro2d and tomcatv. ${ }^{1}$ Retrofitting these 15 instructions into an existing ISA should be feasible; for example, all can be added to the DEC Alpha ISA, without negatively impacting instruction decoding.

5. Free Opcode/Mask augments Free Opcode by generating a Free Mask instruction at the end of each trace. This hybrid scheme addresses register last uses in instructions that are not covered by our particular choice of instructions for Free Opcode.

Current renaming hardware provides mechanisms for register deallocation (i.e., returning physical registers to the free register list) and can perform many deallocations each cycle. For example, the Alpha 21264 deallocates up to 13 registers each cycle to handle multiple 


\begin{tabular}{|l|l||l|l|}
\hline \multicolumn{2}{|c||}{ Integer } & \multicolumn{2}{c|}{ FP } \\
\hline \multicolumn{1}{|c|}{ Opcode } & \multicolumn{1}{c|}{ Operand } & Opcode & Operand \\
\hline addl & 1 & addt & 1 \\
\hline subl & 1 & subt & 1 \\
\hline mull & 1 & mult & 1,2 \\
\hline stl & 2 & stt & 1 , both \\
\hline beq & 1 & fcmov & 1, both \\
\hline lda & 1 & & \\
\hline ldl & 1 & & \\
\cline { 1 - 2 } & & &
\end{tabular}

Table 6: The opcodes used in Free Opcode. Note that for mult, stt, and fcmov, two new versions of each must be added. The versions specify whether the first, second, or both operands are last uses.

instruction retirement or squashing. All five proposed register deallocation techniques use a similar mechanism. Free Mask is slightly more complex, because it can specify up to 32 registers; in this case deallocation could take multiple cycles if necessary. (In our experiments, however, only 7.2 registers, on average, were freed by each mask.)

The five register deallocation schemes are compared in Figure 6, which charts their speedup versus no explicit register deallocation. The Free Register Bit bars show that register deallocation can (potentially) improve performance significantly for small register files (77\% on average, but ranging as high as 195\%). The Free Register Bit results highlight the most attractive outcome of register deallocation: by improving register utilization, an SMT processor with small register files can achieve large register file performance, as shown in Figure 7 . The significance of this becomes apparent in the context of conventional register file design. Singlethreaded, out-of-order processors often double their registers to support greater degrees of parallelism (e.g., the R10000 has 64 physical registers, the 21264 has 80). With multiple register contexts, an SMT processor need not double its architectural registers if they are effectively shared. Our results show that an 8-context SMT with an FSR register file (i.e., support for deallocating registers in idle contexts) needs only 96 additional registers to alleviate physical register pressure, lowering the renaming register cost to $27 \%$ of the ISA-defined registers. Compiler-directed register deallocation for active contexts drops the overhead even further, to only 8 registers or $3 \%$ of the architectural register state.

The Free Register and Free Mask results highlight the trade-off between these two alternative schemes. Free Register is more effective at reducing the number of dead registers, because it deallocates them more promptly, at their last uses. When registers are a severe bottleneck, as in applu, hydro2d, tomcatv, and radix with small register files, Free Register outperforms Free Register Mask. Free Register Mask, on the other hand, incurs less instruction overhead; therefore it is preferable with larger register files and applications with low register usage.

Free Opcode and its variant, Free Opcode/Mask, ${ }^{1}$ are the schemes of choice. They strike a balance between Free Register and Free Mask by promptly deallocating registers, while avoiding instruction overhead. When registers were at a premium, Free Opcode(/Mask) achieved or exceeded the performance of Free Register; with the larger register file and for applications with low register usage, Free Mask performance was attained or surpassed.

For most programs (all register set sizes and both cache hierarchies) Free Opcode(/Mask) met or came close to the optimal performance of Free Register Bit. (For example, it was within 


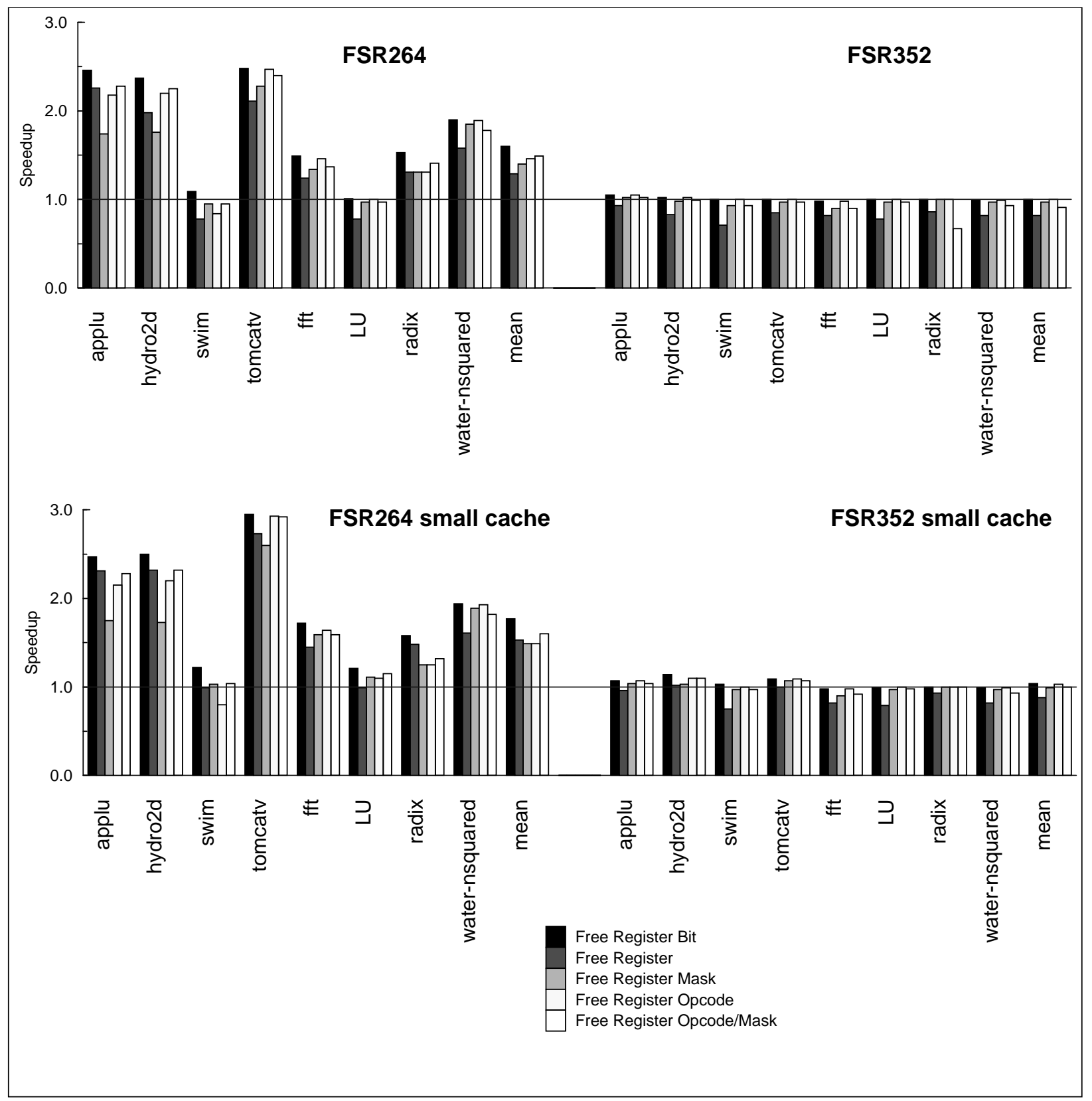

$4 \%$ on average for 264 registers, and $10 \%$ for 352, on the small cache hierarchy.) With further tuning of opcode selection and the use of other hybrid schemes (perhaps judiciously combining Free Opcode, Free Mask, and Free Register), we expect that the gap between it and Free Register Bit will be narrowed even further, and that we will achieve the upper bound of 


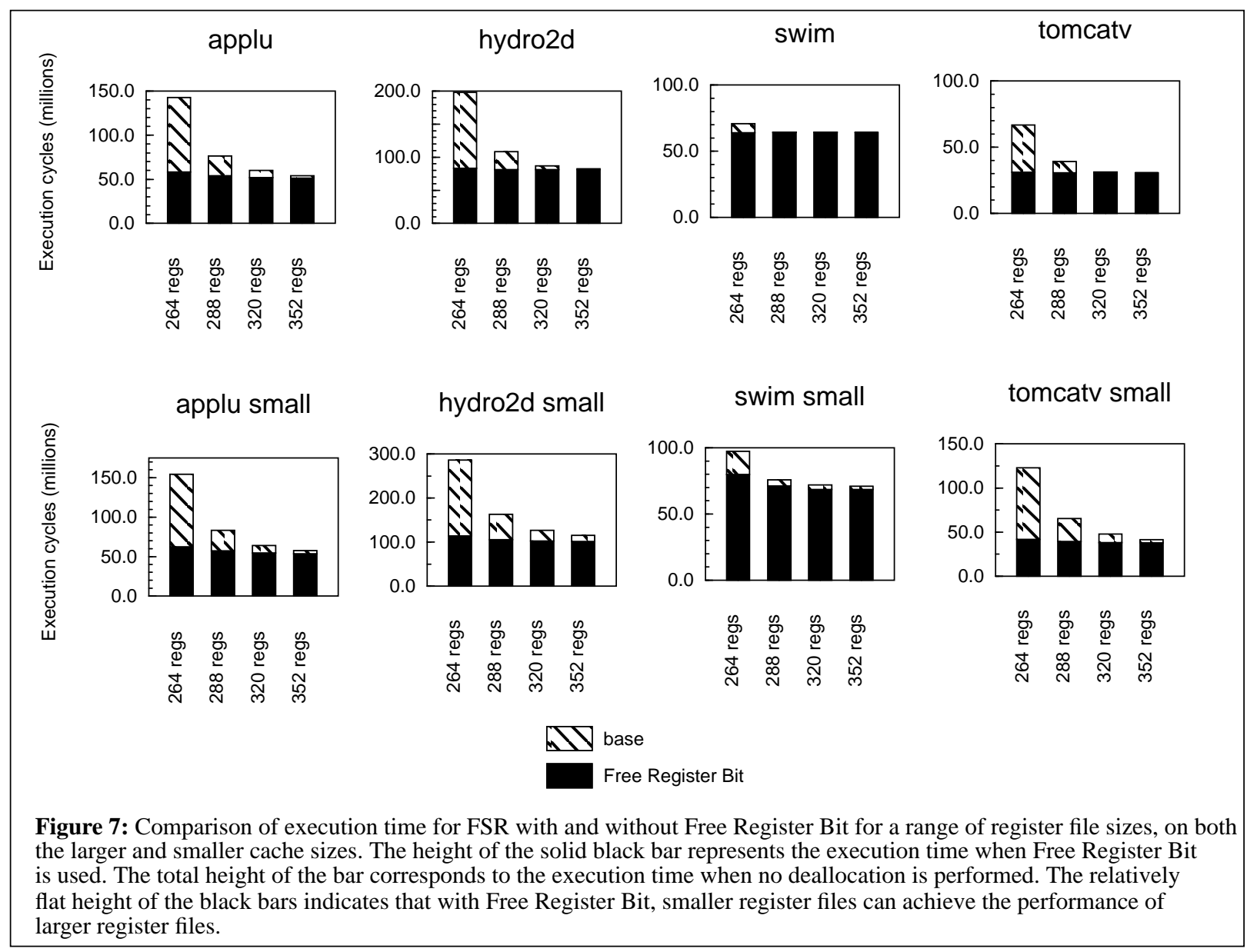

compiler-directed register deallocation performance.

In summary, by providing the hardware with explicit information about register lifetimes, compiler-directed register deallocation can significantly improve performance on small SMT register files, so that they become a viable alternative even with register-intensive applications. Although particularly well-suited for SMT, register deallocation should benefit any out-of-order processor with explicit register renaming.

\section{Related work}

Several researchers have investigated register file issues similar to those discussed in this paper. Large register files are a concern for both multithreaded architectures and processors with register windows. Waldspurger and Weihl [25] proposed compiler and runtime support for managing multiple register sets in a register file. The compiler tries to identify an optimal number of registers for each thread, and generates code using that number. The runtime system then tries to dynamically pack the register sets from all active threads into the register file. Nuth and Dally's [17] named state register file caches register values by dynamically mapping active registers to a small, fast set of registers, while backing the full register name space in memory.

To reduce the required chip area in processors with register windows, Sun designed 3-D register files [22]. Because only one register window can be active at any time, the density of the register file can be increased by overlaying multiple register cells so that they share wires.

Several papers have investigated register lifetimes and other register issues. Farkas, et al. [6] 
compared the register file requirements for precise and imprecise interrupts and their effects on the number of registers needed to support parallelism in an out-of-order processor. They also characterized the lifetime of register values, by identifying the number of live register values present in various stages of the renaming process.

Franklin and Sohi [7] and Lozano and Gao [15] found that register values have short lifetimes, and often do not need to be committed to the register file. Both proposed compiler support to identify last uses and architectural mechanisms to allow the hardware to ignore writes to reduce register file traffic and the number of write ports, but neither applied these concepts to register deallocation. Pleszkun and Sohi [18] proposed a mechanism for exposing the reorder buffer to the compiler, so that it could generate better schedules and provide speculative execution. Sprangle and Patt [21] proposed a statically-defined tag ISA that exposes register renaming to the compiler and relies on basic blocks as the atomic units of work. Part of the register file is used for storing basic block effects, and the rest handles values that are live across basic block boundaries.

Janssen and Corporaal [10], Capitanio, et al. [3], Llosa, et al. [12], Multiflow [4], and Kiyohara, et al. [11] also investigated techniques for handling large register files, including partitioning, limited connectivity, replication, and the use of new opcodes to address an extended register file.

\section{Conclusions}

Simultaneous multithreading has the potential to significantly increase processor utilization on wide-issue out-of-order processors, by permitting multiple threads to issue instructions to the processor's functional units within a single cycle. As a consequence, SMT requires a large register file to support the multiple thread contexts. This raises a difficult design tradeoff, because large register files can consume die area and impact performance.

This paper has introduced new software-directed techniques that increase utilization of the registers in an SMT. Fundamental to these techniques is the global sharing of registers among threads, both for architectural register and renaming register needs. By introducing new instructions or additional fields in the ISA, we allow the operating system and compiler to signal physical register deallocation to the processor, thereby greatly decreasing register waste. The result is more effective register use, permitting either a reduction in register file size or an increase in performance for a given file size.

We have introduced explicit software-directed deallocation in two situations. First, when a context becomes idle, the operating system can indicate that the idle context's physical registers can be deallocated. This permits those registers to be freed in order to serve the renaming needs of other executing threads. Our results show that such notification can significantly boost performance for the remaining threads, e.g., a register file with 264 registers demonstrates performance equivalent to a 352-register file when only 4 threads are running. Second, by allowing the compiler to signal the last use of a register, the processor need not wait for a redefinition of that register in order to reuse it. We proposed several mechanisms for signalling last register use, and showed that on small register files, average speedups of 1.6 can be obtained by using the most efficient of these mechanisms. While our results are shown in the context of an SMT processor, these mechanisms would be appropriate for any processor using register renaming for out-of-order instruction issue. 


\section{References}

[1] R. Alverson, D. Callahan, D. Cummings, B. Koblenz, A. Porterfield, and B. Smith. The Tera computer system. In International Conference on Supercomputing, pages 1-6, June 1990.

[2] J. Boyle, R. Butler, T. Diaz, B. Glickfeld, E. Lusk, R. Overbeek, J. Patterson, and R. Stevens. Portable Programs for Parallel Processors. Holt, Rinehart, and Winston, Inc., 1987.

[3] A. Capitanio, N. Dutt, and A. Nicolau. Partitioned register files for VLIWs: A preliminary analysis of tradeoffs. In 25th International Symposium on Microarchitecture, pages 292-300, December 1992.

[4] R. P. Colwell, R. P. Nix, J. J. O’Donnell, D. Papworth, and P. Rodman. A VLIW architecture for a trace scheduling compiler. IEEE Transactions on Computers, C-37(8):967-979, August 1988.

[5] S. J. Eggers, J. S. Emer, H. M. Levy, J. L. Lo, R. L. Stamm, and D. M. Tullsen. Simultaneous multithreading: A platform for next-generation processors. In IEEE Micro, pages 12-19, October 1997.

[6] K. I. Farkas, N. P. Jouppi, and P. Chow. Register file design considerations in dynamically scheduled processors. In 2nd Annual International Symposium on High-Performance Computer Architecture, pages 40-51, January 1996.

[7] M. Franklin and G. Sohi. Register traffic analysis for streamlining inter-operation communication in finegrained parallel processors. In 25th International Symposium on Microarchitecture, pages 236-245, December 1992.

[8] L. Gwennap. Digital 21264 sets new standard. Microprocessor Report, pages 11-16, October 1996.

[9] M. W. Hall, J. M. Anderson, S. P. Amarasinghe, B. R. Murphy, S.-W. Liao, E. Bugnion, and M. S. Lam. Maximizing multiprocessor performance with the SUIF compiler. IEEE Computer, 29(12):84-89, December 1996.

[10] J. Janssen and H. Corporaal. Partitioned register files for TTAs. In 28th International Symposium on Microarchitecture, pages 303-312, December 1995.

[11] T. Kiyohara, S. Mahlke, W. Chen, R. Bringmann, R. Hank, S. Anik, and W.W. Hwu. Register connection: A new approach to adding registers into instruction set architectures. In 20th Annual International Symposium on Computer Architecture, pages 247-256, May 1993.

[12] J. Llosa, M. Valero, and E. Ayguade. Non-consistent dual register files to reduce register pressure. In 1st Annual International Symposium on High-Performance Computer Architecture, pages 22-31, January 1995.

[13] J. L. Lo, S. J. Eggers, J. S. Emer, H. M. Levy, R. L. Stamm, and D. M. Tullsen. Converting thread-level parallelism to instruction-level parallelism via simultaneous multithreading. In ACM Transactions on Computer and Systems, 15(3):322-354, August 1997.

[14] P. G. Lowney, S. M. Freudenberger, T. J. Karzes, W. Lichtenstein, R. P. Nix, J. S. O’Donnell, and J. C. Ruttenberg. The Multiflow trace scheduling compiler. The Journal of Supercomputing, 7(1/2):51-142, May 1993.

[15] C. L. Lozano and G. Gao. Exploiting short-lived variables in superscalar processors. In 28th International Symposium on Microarchitecture, pages 292-302, December 1995.

[16] S. McFarling. Combining branch predictors. Technical Report TN-36, DEC-WRL, June 1993.

[17] P. R. Nuth and W. J. Dally. The named-state register file: Implementation and performance. In 1st Annual International Symposium on High-Performance Computer Architecture, pages 4-13, January 1995.

[18] A. R. Pleszkun and G. S. Sohi. The performance potential of multiple functional unit processors. In 15th Annual International Symposium on Computer Architecture, pages 37-44, May 1988.

[19] J. P. Singh, J. L. Hennessy, and A. Gupta. Scaling parallel programs for multiprocessors: Methodology and examples. IEEE Computer, 27(7):42-50, July 1993.

[20] Standard Performance Evaluation Council. SPEC CPU '95 Technical Manual. August 1995.

[21] E. Sprangle and Y. Patt. Facilitating superscalar processing via a combined static/dynamic register renaming scheme. In 27th International Symposium on Microarchitecture, pages 143-147, December 1994.

[22] M. Tremblay, B. Joy, and K. Shin. A three dimensional register file for superscalar processors. In Hawaii International Conference on System Sciences, pages 191-201, January 1995. 
[23] D. M. Tullsen, S. J. Eggers, J. S. Emer, H. M. Levy, J. L. Lo, and R. L. Stamm. Exploiting choice: Instruction fetch and issue on an implementable simultaneous multithreading processor. In 23rd Annual International Symposium on Computer Architecture, pages 191-202, May 1996.

[24] D. M. Tullsen, S. J. Eggers, and H. M. Levy. Simultaneous multithreading: Maximizing on-chip parallelism. In 22nd Annual International Symposium on Computer Architecture, pages 392-403, June 1995.

[25] C. A. Waldspurger and W. E. Weihl. Register relocation: Flexible contexts for multithreading. In 20th Annual International Symposium on Computer Architecture, pages 120-129, May 1993.

[26] S. C. Woo, M. Ohara, E. Torrie, J. P. Singh, and A. Gupta. The SPLASH-2 programs: Characterization and methodological considerations. In 22nd Annual International Symposium on Computer Architecture, pages 24-36, June 1995.

[27] K. C. Yeager. The MIPS R10000 superscalar microprocessor. IEEE Micro, pages 28-40, April 1996. 